\title{
A retrospective review of sleep-disordered breathing, hypertenstion and cardiovascular diseases in spinal cord injury patients
}

\author{
Spinal Cord (2015) 53, 496-497; doi:10.1038/sc.2015.16; published online 10 February 2015
}

We read with great interest the article by Goh et al. ${ }^{1}$ on ambulatory blood pressure (BP) monitoring and diurnal urine production in 44 tetraplegic and 10 paraplegic spinal cord injury (SCI) patients. Data were obtained retrospectively to quantify diurnal BP patterns including nocturnal hypertension and to measure diurnal urine production. The authors concluded that 'ambulatory BP monitoring in patients with SCI and clinically significant BP disorders detected a high incidence of reversed dipping and nocturnal hypertension.' In addition, the authors speculated that 'elevated nocturnal BP may contribute to nocturnal diuresis that might cause relative volume depletion and thereby contribute to daytime orthostatic hypotension.' After reading these conclusions and on the basis of available evidence of very high prevalence of sleep-disordered breathing (SDB) and cardiovascular morbidities in SCI patients, we felt compelled to write this letter. Our major concern is that the authors do not report information on sleeprelated disorders as contributors to the mechanism of nocturnal hypertension in SCI patients. Further, new data (summarized below) suggest that under-recognition of SDB could contribute to the increased prevalence of hypertension and cardiovascular disorders in this population of patients. In fact, more than half of patients with SCI are obese or overweight, leading to an increased risk for both SDB and cardiac diseases. ${ }^{2}$

Our group has been studying SDB among SCI patients, and, we have found that SDB, defined by the apnea-hypopnea index $\geqslant 5$ events per hour, was present in $77 \%$ of chronic SCI patients, with rates higher among those with cervical compared with thoracic injuries (93\% vs 55\%, respectively, $P<0.05$ ). ${ }^{3}$ One in four cervical SCI had Cheyne-Stokes respiration pattern during their overnight laboratory sleep study, and one in five had hypertension or cardiac disease. Furthermore, nearly all patients had poor sleep quality and daytime sleepiness or fatigue as measured by the Pittsburgh Sleep Quality Index, the Fatigue Severity Scale and the Epworth Sleepiness Scale. In the manuscript, the authors did not report information on whether or not patients had SDB or whether other cardiovascular comorbidities were present.

We also are interested in the rates of diagnosis of SDB (obstructive sleep apnea and/or central sleep apnea) in SCI patients receiving usual clinical care, and, to that end, we reviewed medical records of patients who were included in the local Spinal Cord Injury and Disorders Outcomes database at the John D Dingell VA Medical Center. We identified a total of 168 veterans with SCI/or disorder. We found that only 37 patients (22\%) were evaluated for SDB and $34(20 \%)$ had SDB diagnosis confirmed by the sleep study, of whom only 6 patients $(18 \%)$ were using the positive airway pressure (PAP) therapy. Moreover, $89(53 \%)$ of SCI veterans had hypertension and $26(16 \%)$ had cardiovascular disease (including one or more of the following diagnoses: myocardial infarction, coronary artery disease and/or chronic heart failure), which may be caused or exacerbated by untreated SDB.

The strong relationship between untreated SDB and nocturnal hypertension had been repeatedly confirmed in ablebodied individuals with SDB. Ambulatory BP monitoring allows accurate assessment of circadian BP changes, which can identify a blunted nocturnal decline in BP and indicate the possible secondary cause of hypertension, such as sleep apnea. Specifically, the nondipping BP phenomenon measured by ambulatory BP monitoring was found in untreated patients with SDB and was associated with poor outcome. Therefore, on the basis of above findings, it is unfortunate that in this manuscript the authors did not assess the presence of SDB in these patients.

We thank Goh et al. for their efforts to address these important problems in SCI patients. However, awareness of sleep disorders as potentially treatable risk factors for nocturnal hypertension and altered diurnal ambulatory BP is critical to achieving the best outcomes for SCI patients. In this vulnerable population, the lack of awareness and treatment of SDB among SCI patients may represent a form of healthcare disparity for the disabled. The consequences of untreated SDB and associated cardiovascular disorders are severe and could contribute to the higher mortality rate in this population. We therefore recommend consideration of SDB as a possible underlying variable explaining the findings reported in this paper.

\section{CONFLICT OF INTEREST}

The authors declare no conflict of interest.

\section{ACKNOWLEDGEMENTS}

Research mentioned in this letter was supported by the United States Department of Veterans Affairs Merit Review Award 1I01CX001040 and Career Development Award 1IK2CX000547 from the Clinical Science Research \& Development Service of the VA Office of Research and Development.

\section{DISCLAIMER}

The opinions expressed in this article reflect those of the authors and do not necessarily reflect official views of the VA.

$$
\begin{array}{r}
\text { A Sankari }{ }^{1,2}, \text { JL Martin }{ }^{3,4} \text { and M Badr }{ }^{1,2} \\
{ }^{1} \text { Department of Medicine, John D Dingell VA Medical Center, } \\
\text { Detroit, MI, USA; } \\
{ }^{2} \text { School of Medicine, Wayne State University, Detroit, MI, USA; }
\end{array}
$$


${ }^{3}$ Geriatric Research Education and Clinical Center, VA Greater Los Angeles Healthcare System, North Hills, CA, USA and ${ }^{4}$ David Geffen School of Medicine at the University of California, Los Angeles, CA, USA E-mail: abdulghani.sankari@va.gov Parts of these findings were presented at the American Professional Sleep Society meeting in Minnesota on June 2014 and reported in abstract form.
1 Goh MY, Wong ECK, Millard MS, Brown DJ, O'Callaghan CJ. A retrospective review of the ambulatory blood pressure patterns and diurnal urine production in subgroups of spinal cord injured patients. Spinal Cord 2015; 53: 49-53.

2 Weaver FM, Collins EG, Kurichi J, Miskevics S, Smith B, Rajan S et al. Prevalence of obesity and high blood pressure in veterans with spinal cord injuries and disorders: a retrospective review. Am J Phys Med Rehabil 2007; 86: 22-29.

3 Sankari A, Bascom A, Oomman S, Badr MS. Sleep disordered breathing in chronic spinal cord injury. J Clin Sleep Med 2014; 10: 65-72. 